\title{
No difference in effectiveness of Hawley and vacuum-formed retainers at 12 months
}

\author{
Abstracted from \\ Barlin S, Smith R, Reed R, Sandy J, Ireland AJ. \\ A retrospective randomized double-blind comparison study of the effectiveness \\ of Hawley vs vacuum-formed retainers. Angle Orthod 2011; 81: 404-409. \\ Address for correspondence: Dr Anthony John Ireland, Child Dental Health, \\ Bristol Dental Hospital, Lower Maudlin Street, Bristol, BS1 2LY UK. E-mail: tony.ireland@bristol.ac.uk
}

\section{Question: Is a vacuum-formed retainer more effective than a Hawley retainer in maintaining arch width and incisor alignment following orthodontic treatment?}

\section{Design Randomised trial.}

Intervention Patients were randomised to either a Hawley or vacuumformed retainer following completion of straight wire fixed appliance therapy. Study models were fabricated for each patient on day of debond and 2 months, 6 months, and 12 months after debond. Outcome measure Models were assessed at each time point for upper and lower intermolar widths, intercanine widths, arch length and a modified Little's index of irregularity. Method error was determined by repeating the measurements on 10 sets of models.

Results There were no statistically significant differences between each of the two retainers for any of the variables under test at any time period.

Conclusions There is no statistical or clinically significant difference in the measured arch width, arch length or modified Little's index over a 12-month period between Hawley and vacuum-formed retainers when worn full-time. Therefore, other factors may be more important when deciding on the more appropriate form of retainer.

\section{Commentary}

This study compares the effectiveness of Hawley and vacuum formed retainers (VFRs) in maintaining specific arch form features after orthodontic treatment. Following completion of orthodontic treatment with fixed appliances, 170 patients were randomly allocated to receive either Hawley or VFRs, and instructed to wear them full-time for 24 hours, except for cleaning. Impressions were taken of participants in both groups 2, 6 and 12 months after debond, and study models cast. The following measurements were recorded in upper and lower arches: incisor irregularity; intercanine width; intermolar width and arch length. No statistically or clinically significant difference between the two groups was found for any of the measurements examined; both types of retainer were found to be equally effective.

Some points are worth discussion. Although the study was set up as a prospective randomised clinical trial, some years passed before the data were analysed. The authors have erred on the side of statistical caution and classified it as a retrospective study. More important, probably, is the fact that the drop-out rate was high: full records for inclusion were only available for 82/170 participants (48\%). This exposes the findings to the risk of attrition bias, and demonstrates the difficulty of conducting clinical trials; maybe just as difficult as getting patients to wear their retainers! Instructing patients to wear their retainers full-time (except for cleaning) for 12 months is rather a cautious, if not onerous, regime. Perhaps those unable to comply were distributed equally between the two groups.

Despite these criticisms, this is a well-designed study that contributes to our knowledge of retention, and helps us to provide patients with advice about these two types of retainer.

VFRs have certainly grown in popularity due to their ease of fabrication, aesthetic advantage and cost effectiveness. Because of studies like this, we can now add clinical effectiveness to that list. It is also one of a number of recent studies that finds little, if any, difference between the effectiveness of various retainer types or wear regimes. It's all good fuel for future Cochrane review updates, even if we only become more certain that the conclusion is inconclusive.

\section{Practice points}

- This study compares the effectiveness of Hawley and vacuum formed retainers in the first year after completion of orthodontic treatment with fixed appliances.

- There was no statistically or clinically significant difference in the effectiveness of these retainers.

Jan Ledvinka

Orthodontist, Sydney, Australia

Evidence-Based Dentistry (2012) 13, 24. doi:10.1038/sj.ebd.6400846 